\title{
Morphometric parameters of cardiac implantable electronic device (CIED) pocket walls observed on device replacement
}

\author{
R. Steckiewicz, E.B. Świętoń, A. Kołodzińska, M. Bogdańska, Ł. Fus, P. Solarz \\ Department of Cardiology, Medical University of Warsaw, Poland
}

[Received: 25 February 2017; Accepted: 27 April 2017]

Background: The final stage of a conventional de-novo cardiac implantable electronic device (CIED) implantation procedure with transvenous lead insertion involves the formation of a pocket by tissue separation superficial to the pectoralis major muscle in the right or left infraclavicular region, where the device is subsequently placed. Over time, a scar "capsule" is formed around the CIED as a result of normal biological remodelling.

Materials and methods: The purpose of this study was to analyse the structure and present the variations of CIED capsules observed during device replacement. The nature and extent of this local tissue remodelling, which had occurred from the time of device implantation to its replacement in 2016 (10 \pm 3.1 years), was analysed in 100 patients (mean age $77.1 \pm 14.5$ years), including 45 women and 55 men.

Results: The most prevalent types of "capsules" (70\% of cases) were those with similar thickness of both walls or a slightly thicker posterior $(<1.0 \mathrm{~mm})$ than anterior wall $(<0.5 \mathrm{~mm})$. The second most common capsule type (23\% of cases) was characterised by a significantly thicker posterior wall of scar tissue $(>1.0 \mathrm{~mm})$. The third group of capsules was characterised by various degrees of wall calcification (7\% of cases).

Conclusions: The extent and nature of scar tissue structure in the CIED pocket walls seem to correlate with the relative position of cardiac lead loops with respect to the device itself; where the more extensive scarring is likely to result from pocket wall irritation in the capsule formation phase due to lead movements underneath the device. The group of cases with calcified capsules was characterised by "old" device pockets (> 13 years) and the oldest population (patients in their 80s and 90s). (Folia Morphol 2017; 76, 4: 675-681)

Key words: pacemaker, implantable cardioverter-defibrillator, capsule, structure

\section{INTRODUCTION}

During most of the current cardiac pacemaker (PM) and implantable cardioverter-defibrillator (ICD), i.e. cardiac implantable electronic device [CIED] implantation procedures, cardiac leads are introduced into the venous system via vessels located in the deltopectoral triangle. In such situations, the CIED is placed in a subcutaneous pocket created superficial to the pectoralis major muscle $[3,10]$. Creating such a pocket involves tissue traumatisation, including 
Table 1. Cardiac implantable electronic device (CIED) types found during device replacement due to elective replacement indication (ERI) (no. of cases); type of arrhythmia detected in electrocardiogram (ECG)/Holter tracings (no. of cases), the period (years) between CIED implantation and replacement with pocket revision (PM vs. ICD, $p<0.001$ ), ERI and CIED replacement, and pacemaker dependency: emergence of intrinsic rhythm with the pacing rate lowered to $30 \mathrm{pulses} / \mathrm{min}$, after medication during the procedure

\begin{tabular}{|c|c|c|c|c|}
\hline $\begin{array}{l}\text { CIED type } \\
\text { (no. of cases) }\end{array}$ & $\begin{array}{l}\text { Arrhythmia type } \\
\text { (no. of cases) }\end{array}$ & $\begin{array}{l}\text { Indwelling period } \\
\text { [years] }\end{array}$ & $\begin{array}{c}\text { ERI } \\
\text { [years] }\end{array}$ & $\begin{array}{l}\text { Device-dependency } \\
\text { (no. of cases) }\end{array}$ \\
\hline PM (83) & ECG/Holter & PM & PM & PM \\
\hline DDD (49) & $\begin{array}{c}\text { TBS (30) } \\
\text { AV block (19) }\end{array}$ & \multirow{3}{*}{$\begin{array}{c}2-24 \text { years } \\
\text { (mean } 10.7 \pm 2.8)\end{array}$} & $\begin{array}{l}\text { DDD: } 6-15 \text { years } \\
\text { (mean } 9.4 \pm 2.4 \text { ) }\end{array}$ & \multirow{3}{*}{$\begin{array}{l}\text { No intrinsic rhythm } \rightarrow \text { total } \\
\text { PM dependency (12) }\end{array}$} \\
\hline VI (29) & $\begin{array}{l}\text { TBS (18) } \\
\text { CAF (11) }\end{array}$ & & $\begin{array}{l}\text { VVl: 7-13 years } \\
\text { (mean } 11.1 \pm 2.9)\end{array}$ & \\
\hline AAl (5) & SSS (5) & & $\begin{array}{l}\text { AAl: } 11-12 \text { years } \\
\text { (mean } 11.4 \pm 0.5)\end{array}$ & \\
\hline ICD (17) & Prevention & ICD VR/DR & ICD & ICD \\
\hline ICD VR (12) & Primary (15) & \multirow{2}{*}{$4-9$ years (mean $6.4 \pm 1.4$ ) } & $\begin{array}{c}\text { VR } 4-9 \text { years } \\
\text { (mean } 6.6 \pm 1.4)\end{array}$ & \multirow{2}{*}{$\begin{array}{l}\text { Predominantly intrinsic } \\
\text { rhythm/occasional pacing }\end{array}$} \\
\hline ICD DR (5) & Secondary (2) & & $\begin{array}{c}\text { DR 4-7 years } \\
\text { (mean } 5.4 \pm 2.7)\end{array}$ & \\
\hline
\end{tabular}

PM — pacemaker; DDD — atrioventricular (dual-chamber) pacemaker; VVI — ventricular pacemaker; AAI — atrial pacemaker; ICD VR — single-chamber implantable cardioverter-defibrillation; ICD DR — dual-chamber implantable cardioverter-defibrillator; SSS — sick sinus syndrome; TBS — tachycardia-bradycardia syndrome; AV block — second or third degree atrioventricular block; CAF — chronic atrial fibrillation

damage to myocytes, nerve fibres, and blood vessels, as well as blood extravasation. In the months following the procedure the histological and morphometric parameters of the tissues surrounding the device undergo gradual remodelling into a fibrotic capsule.

The course of CIED implantation procedure and the time period after device implantation have, it seems, a significant effect on the extent of tissue remodelling. Moreover, the type of device and leads implanted may affect the nature and rate of structural and morphometric remodelling $[1,9]$.

Typically, it is the lead placement inside the heart or vessels that has been analysed in terms of correlations between fibrous tissue formation and its consequences [5]. Also, a vast majority of literature reports on the histomorphology of CIED pockets focus on secondary pacing system infections and their sequelae [11]. The purpose of this paper, however, was to visually present and analyse morphohistologic parameters of CIED pockets in non-complicated cases. Assessments of the nature of tissue remodelling that had taken place from device implantation to its replacement was presented based on our own observations.

\section{MATERIALS AND METHODS}

A total of 100 consecutive CIED (17 ICDs and 83 PMs) replacement procedures conducted by the same operating personnel at our centre between January 1, 2016, and October 21, 2016 were included in the study (Table 1).

These procedures were conducted in 45 females aged 30-98 (mean $80.1 \pm 12.6$ ) years (during device replacement) and 55 males aged 41-95 (mean $75.9 \pm 11.9$ ) years (during device replacement).

Each procedure had been dictated by the device having reached the elective replacement indication phase due to projected battery depletion. Conditions that could affect typical capsule formation, such as: infections, skin lesions, too superficial subcutaneous position of the CIED, etc., were excluded from analysis. Neither did we analyse cases following procedures such as device revision, device up-grade, repair procedures, or other procedures whose timing and/or extent could considerably affect device pocket remodelling.

The analysed procedures were characterised by normal device follow-up interrogation values from the time of device implantation to its replacement due to elective replacement indication.

The device pockets were assessed in terms of their morphometric parameters and the type of "capsuleforming" tissue surrounding the CIED. We distinguished three distinct CIED pocket forms (in terms of their anterior and posterior wall evaluation) based on the following criteria: 


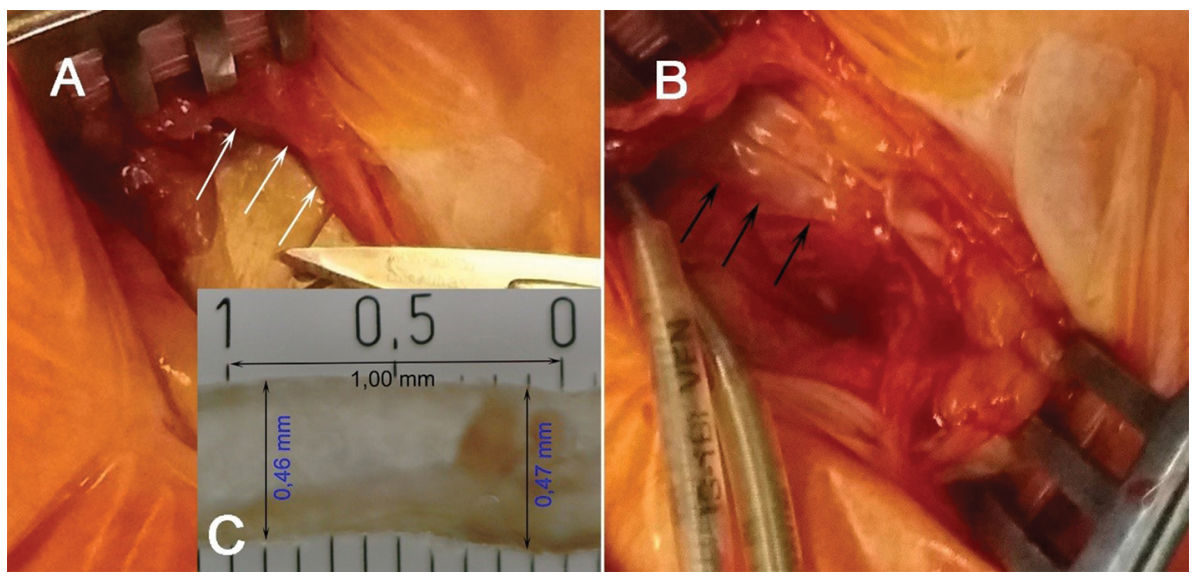

Figure 1. A-C. Cardiac implantable electronic device (CIED) pocket in an 86-year-old man, during a second device replacement procedure 13 years after implantation of a DDD-mode pacemaker (Talos DR, Biotronik). The leads are arranged around the CIED margin: an atrial lead (SX-53 JBP, Biotronik) and a ventricular lead (CapSure SP Novus, Medtronic); A. Edge of a transected anterior wall of the device capsule (white arrows); B. Wrinkled posterior wall of the capsule (black arrows); C. Measure of anterior capsule wall thickness (220-fold magnification).

- Group I - "typical" pockets, with a thin and flexible layer of scar tissue forming the anterior $(<0.5 \mathrm{~mm})$ and/or the posterior $(<1.0 \mathrm{~mm})$ wall;

- Group IIA - pockets with a "typical" anterior wall (see above) and the posterior wall characterised by localised scar tissue thickening (> 1-2 mm);

- Group IIB - pockets with a "typical" anterior wall (see above) and the (usually entire) posterior wall formed of massive scar tissue (> 1-2 $\mathrm{mm}$ );

- Group III - pockets with partial/focal or total wall calcification.

Intraoperative qualification of individual cases into one of these specific groups has been illustrated in the figures of characteristic types of CIED "capsules" included in this paper.

All CIED replacement procedures were conducted via conventional techniques, with local anaesthesia. After opening the device pocket, a visual inspection and palpation of its walls were conducted to assess, among others, the positions of the leads with respect to the device itself. After ascertaining normal lead function, supported by device interrogation findings, a new device was inserted and connected. In order to avoid any potential damage to the lead insulation layer during tissue dissection, in some cases a control fluoroscopy (posteroanterior view; OEC 9900 Elite fluoroscopy system, GE) was conducted immediately prior to the procedure.

In patients that were representative for the selected group types, biopsy samples (up to $2 \times 4 \mathrm{~mm}$ ) of the pocket wall were collected from the site of its incision, towards the end of the procedure, prior to device pocket closure. Digital microscopy images of collected samples were recorded and linear measurements were made off-line with respect to a $1 / 10 \mathrm{~mm}$ $(100 \mu \mathrm{m})$ micrometric reference scale (Fig. 1C).

The tissue samples were fixed in $4 \%$ buffered formalin, embedded in paraffin blocks, and sectioned into 4-micrometer sections, deparaffinised and stained with haematoxylin-eosin (H\&E) and the Masson's trichrome method.

Data on cardiac lead diameter included in figure captions also help visually assess the amount of the surrounding scar tissue. Lead diameter reference scale: 1 French unit $(1 \mathrm{~F})=0.3333 \mathrm{~mm}=0.0131 \mathrm{in}$.

Our statistical analysis used numerical variables in the form of mean values, standard deviations, and statistical significance ( $p$-values).

This study had been approved by the Institutional Review Board.

\section{RESULTS}

Device pocket structure was analysed over 100 consecutive CIED replacement procedures conducted in the analysed time period, which yielded the final sample size of: 83 cases of PM pockets in patients aged from 30 to 98 years (mean $79 \pm 12.8$ ) and 17 cases of ICD pockets in patients aged from 63 to 82 years (mean $72 \pm 6.8$ ) (mean age difference between groups: $p=0.0017$ ). The time period from device pocket formation to the assessment of the resulting capsule in 2016, ranged from 2 to 24 years (mean $10.7 \pm 2.8$ ) for PM replacement, and from 4 to 9 years (mean $6.4 \pm 1.4$ ) for ICD replacement 


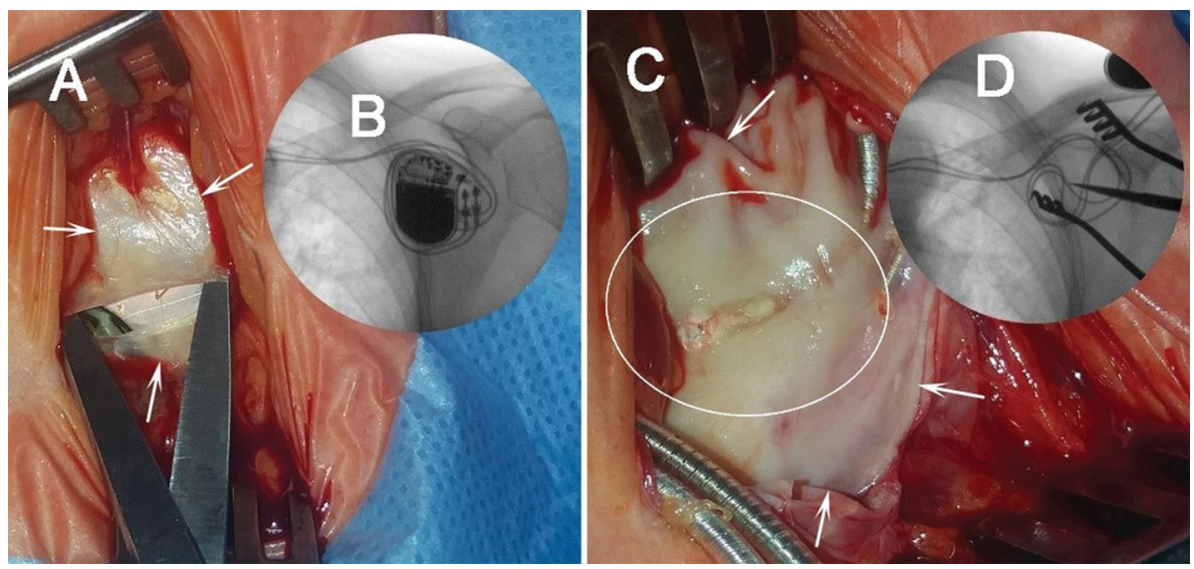

Figure 2. A-D. Female, 74 years old, first-time pacemaker (PM) (Axios DR, Biotronic) replacement 10 years after implantation; A. Anterior wall of the capsule covering the PM after opening the device pocket (arrows); B. Fluoroscopic image: cardiac lead position with respect to the PM immediately adjacent to, and a short distance away from, the device; $\mathbf{C}$. The posterior wall of the capsule, with its peripheral structure similar to that of the anterior wall (arrows), centrally located focal thickening of scar tissue surrounding a lead segment (CapSure SP Novu, 6 F, Medtronic) located immediately underneath the PM (white oval); D. Fluoroscopic image: lead position following PM removal.
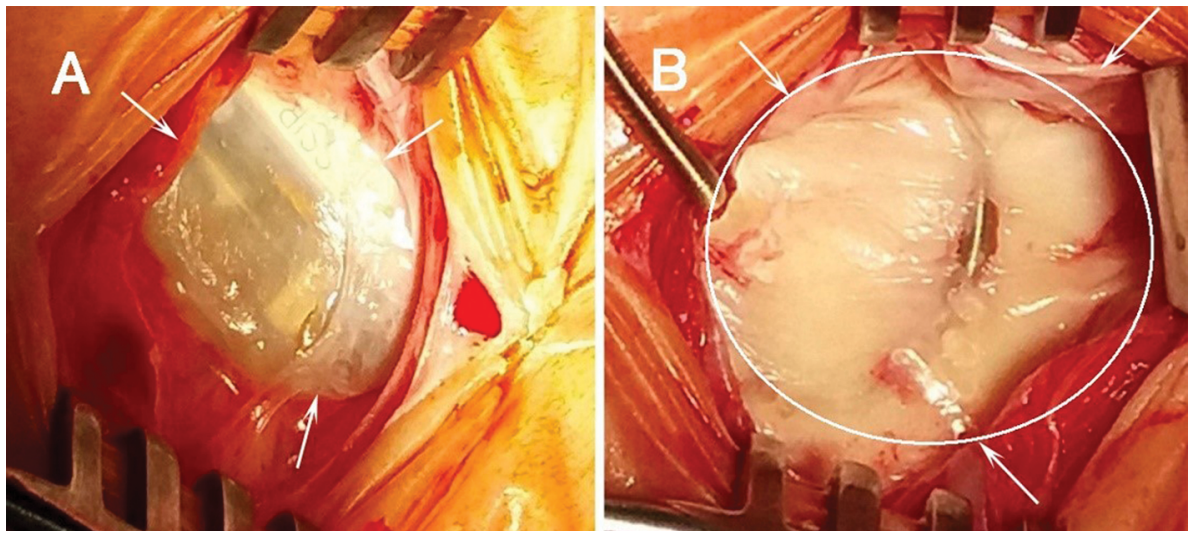

Figure 3. A, B. Female, 88 years old; pocket walls during second-time SSI-mode pacemaker (PM) replacement (2016) 19 years after a single-chamber PM implantation; A. Thin, typical layer of scar tissue forming the anterior wall of the PM capsule (arrows); B. Massive layer of scar tissue forming the posterior capsule wall, with an encased cardiac lead loop (oval) (TiR 60-BP, lead diameter $2.2 \mathrm{~mm}$, Biotronik) visible.

procedures. The difference in the mean time period between the two types of CIEDs: $p<0.001$.

The proportions of the device pocket group types based on the structure of their anterior and posterior walls, were as follows:

- Group I- $70 \%$ of device capsules were characterised by a thin, flexible layer of scar tissue forming the anterior and posterior walls of the pocket; this was the most common morphometric finding. The tissue of the "capsule" could be easily separated from the device and/or leads (example: Fig. 1).

- Subgroup IIA - $18 \%$ of capsules were characterised by anterior wall structure similar to that specified for group I, i.e. a thin, flexible layer of scar tissue, while the posterior wall was characterised by localised thickening of tissue surrounding the leads located underneath the CIED (example: Fig. 2).
- Subgroup IIB - $5 \%$ of capsules; it was not quite as common a finding in terms of posterior pocket wall characteristics: flat, massive scar tissue of increased density, whose surface area typically corresponded to the size of the PM. The anterior wall was usually visibly different from a typically structured pocket wall (see above) (example: Fig. 3).

- Group III - 7\% of capsules were distinctly different from the other capsule types: they were characterised by a hard, calcified structure, closely adherent to the CIED, which usually had to be manually broken during device replacement, and whose edges were sharp. Among the cases analysed here there were those with the entire device capsule being calcified, as well as those with focal wall calcifications (example: Fig. 4). 

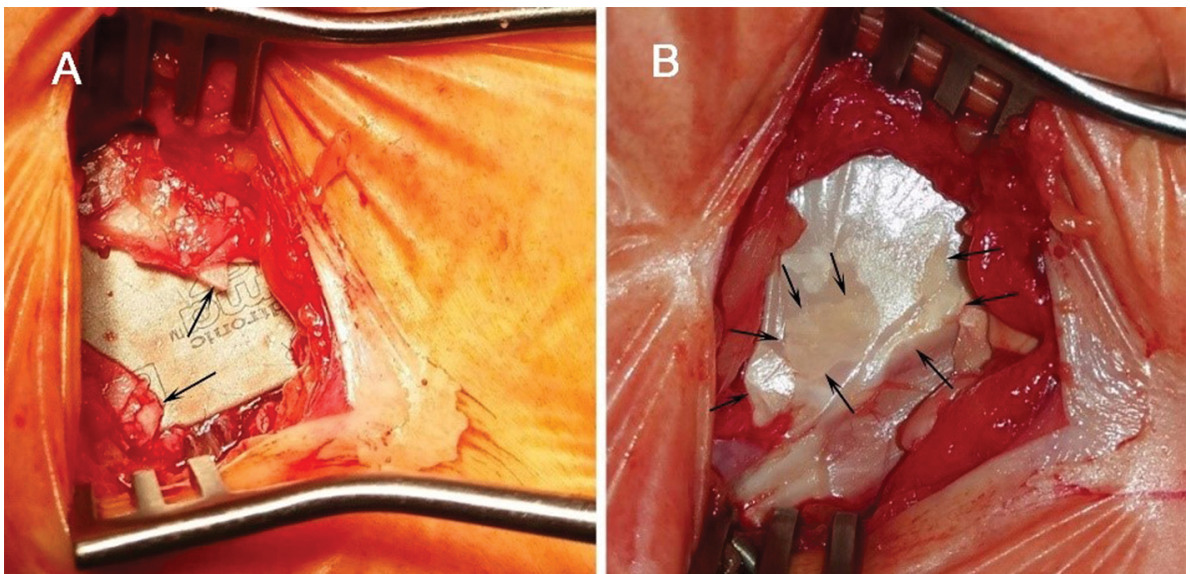

Figure 4. A, B. Male, 88 years old, an 18-year-old device pocket with evidence of capsule wall calcification, during a second-time cardiac implantable electronic device (CIED) replacement (2016). Leads (SX-JBP Synox and TiR 60-BP, Biotronik) are positioned marginally around the pacemaker; A. The entire anterior capsule wall is in the form of a hard calcified plate. Sharp edges of the capsule following its transection (arrows); B. Posterior wall: visible calcified "islets" of a lighter colour with respect to other areas of the pocket bed (arrows).
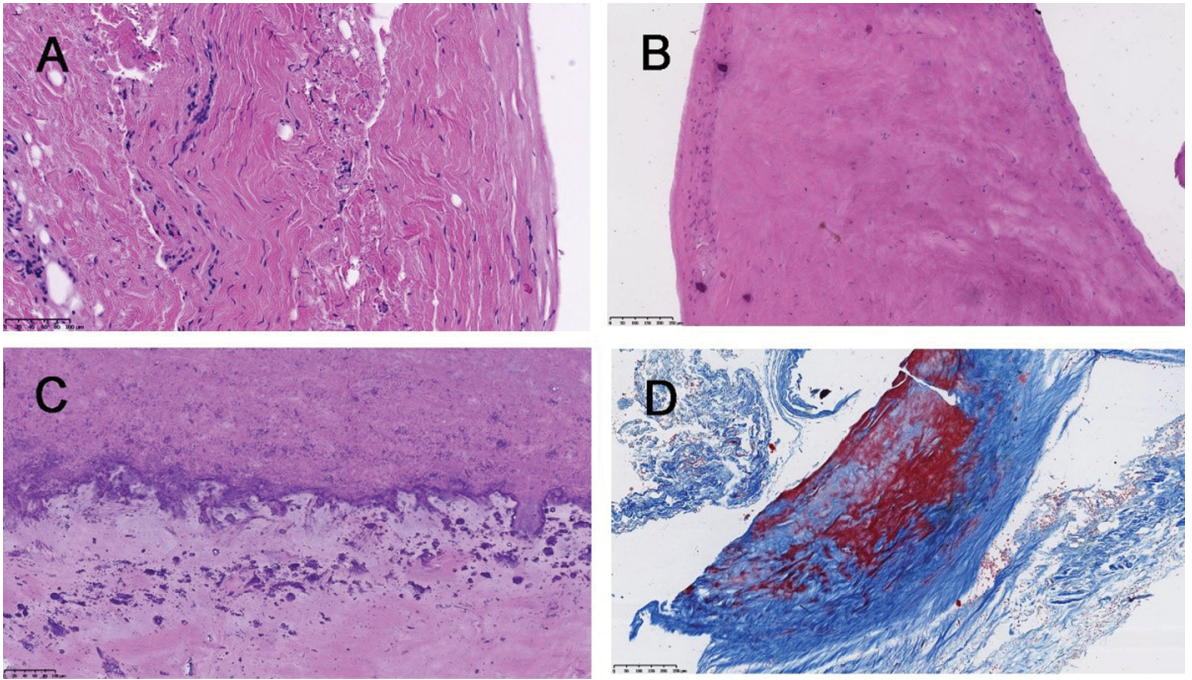

Figure 5. A-D. Histopathological image of cardiac implantable electronic device (CIED) capsules (Figs. 3-5); A. Cell-poor fibrous connective tissue. Small clusters of mononuclear cells and numerous vessels are visible, especially in "loose" connective tissue; B. Partly hyalinised fibrous connective tissue with sparse cells and occasional foci of calcification; C. Partly hyalinised fibrous connective tissue undergoing calcification along the fibres; D. Masson's (trichrome) staining revealed fibrous connective tissue fibre atrophy and calcification along connective tissue fibres.
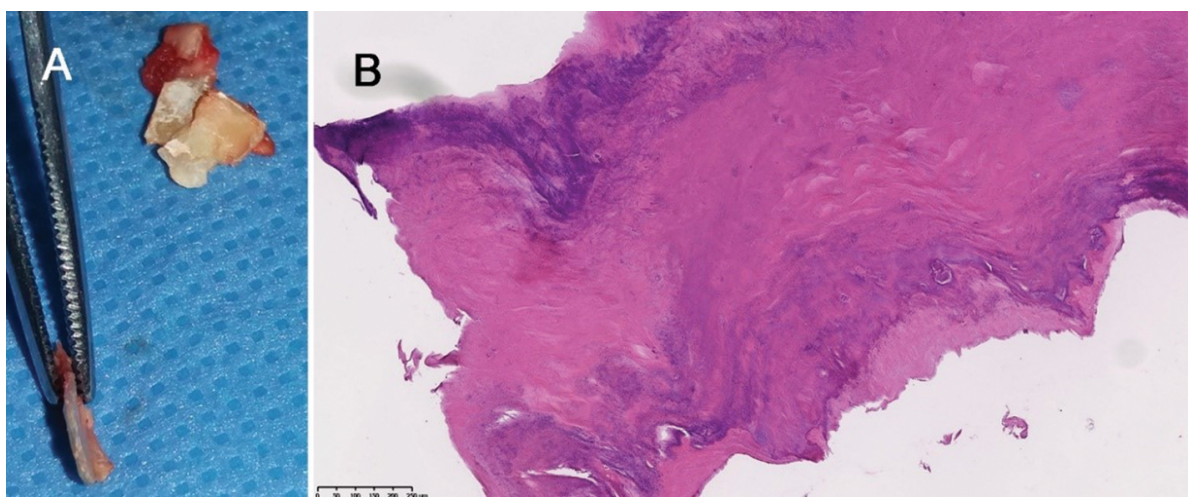

Figure 6. A. Parts of a calcified capsule wall; B. Completely calcified fragment of a device pocket. No collagen fibres, no cellular structures. Haematoxylin-eosin staining; magnification $\times 100$. 
No evidence of capsule calcification was observed in PM patients under 80 years of age. Group III capsule characteristics were observed in 7 octogenarians (mean age 88.5 years); these cases constituted $14 \%$ of all patients in their $80 \mathrm{~s}(\mathrm{p}=0.019)$. The presence of pocket calcification also correlated with the "age" of CIED pockets ( $>13$ years; mean $13.5 \pm 0.8$ ).

Histopathological evaluation showed capsule variety (Figs. 1-3), which was seen in a vast majority of Group I and II patients. Capsule wall structure was cell-poor fibrous connective tissue with mononuclear cells and a large number of vessels, especially within fibrous connective tissue. In Group III, we observed focal (Fig. 5 C, D)/planar (Figs. 4, 6) hyalinisation of the fibrous tissue with calcification along the fibres.

\section{DISCUSSION}

The final stage of a de-novo CIED implantation procedure with transvenous lead insertion involves the formation of a subcutaneous pocket in the right or left infraclavicular region, where the implanted device is subsequently placed. Pocket formation requires mechanical separation of subcutaneous tissues, typically superficial to the pectoralis major muscle, in an area similar to the size of the device being implanted. A considerable disproportion in this respect, i.e. the size of the pocket being much larger than necessary, increases the risk of a haematoma or Twiddler's syndrome $[4,6]$.

Formation of a "capsule" around the CIED is the final stage of normal biological remodelling of tissues damaged during pocket formation. It consists of these gradual and overlapping phases: inflammatory phase and the phase of scar tissue formation and remodelling. Following an injury, the damaged tissue is gradually replaced with new granulation tissue. The intercellular matrix is replaced with collagen, whose haphazardly arranged fibres become organised. The cell-rich granulation tissue gradually transforms into cell-poor scar tissue $[7,12,13]$. The final stage involves the formation of a scar tissue layer, closely adhering to the CIED.

The cases analysed here showed the largest proportion of "capsules" in the form of a thin, flexible layer of scar tissue surrounding the whole CIED and those with a slightly thicker posterior than the anterior wall. Due to its high prevalence, this capsule type (group) was described as typical, with its characteristic flexibility of the scar tissue. If device replacement required adapting the pocket size to the slightly different shape of the new CIED, the capsule was easy to detach from the adjacent tissues and from the device. This type of device pocket was usually accompanied by the extravascular lead segments positioned marginally to the device being replaced, with lead loops lying in the same plane as the CIED, neither deeper nor more superficial.

The second most common pocket type was characterised by thicker scar tissue underneath the device, which was accompanied by lead positioning underneath the PM. Whenever the lead loops had been touching the surface of the CIED either focally or with a short segment of their extravascular course, only a localised scar capsule thickening was observed immediately around the lead, with a typical capsule structure in other areas. When long loops of cardiac leads had been positioned in one plane underneath the CIED, we observed the resulting formation of a plate-like mass of hard scar tissue in that plane, sometimes encasing the device itself.

A less common CIED capsule variation consisted of capsules with evidence of focal or generalised wall calcification. Adapting the existing pocket to a new device of a slightly different shape required breaking the hardened capsule walls, with the calcified fragments exhibiting sharp edges. This type of capsule demanded particular attention during CIED replacement in one patient with chronic hepatitis $\mathrm{C}$, due to the risk of potential transmission of the pathogen to the operating personnel. Pocket calcification may also predispose to lead insulation damage, which is of particular significance in pacemaker-dependent patients.

During CIED implantation procedures the operator usually arranges the leads around the device. However, in some cases lead loops can spontaneously migrate to be superficial or deep to the device, which results in a more pronounced scar tissue formation. A "loose" pocket in the early stage of capsule formation facilitates movements of extravascular lead segments and irritation of healing tissues, which, in conjunction with the blood cells accumulating there, may affect the extent and shape of the forming scar tissue.

The components of cardiac lead insulation (polyurethane, silicon rubber) are considered to be compatible with the surrounding biological environment, which most likely eliminates them as a potential cause of the localised scarring observed in this study [9]. 
Pacemaker capsule calcification, in this study observed in patients in their 80s and 90s, with an over 13-year-old device pocket. Capsule calcification was not observed in the case of ICD pockets. However, the latter type of CIEDs was found in younger patients with shorter time periods to device replacement, for reasons including indications for this type of electrotherapy and the devices' individual use of power.

Formation of massive scar tissue in the pocket walls and/or wall calcification make it more difficult to isolate leads during device removal as well as to implant new leads during device up-date procedures $[2,8]$. This mechanism of potential lead insulation damage becomes especially important in patients without an intrinsic rhythm, in whom loss of pacing effectiveness may become immediately life-threatening.

\section{Limitations of the study}

For the purposes of this manuscript, the sample size was limited to 100 cases, which the authors believe to be a representative sample for this type of analysis. As the most commonly observed anterior and posterior capsule wall thickness was below $0.5 \mathrm{~mm}$ and $1.0 \mathrm{~mm}$, respectively, these values were selected as threshold values differentiating the "normal" and "thickened" scar tissue layers.

\section{CONCLUSIONS}

Morphometric parameters of a formed CIED capsule are significantly affected by spatial relations between the device and the extravascular cardiac lead segments, where lead loop migration underneath the device may facilitate scar tissue formation in the posterior wall of the device pocket.

The cases of pocket calcification discovered in our patients showed this phenomenon occurring exclusively in the oldest patient group in conjunction with "old" device pockets, although calcification was not present in all such cases.

\section{REFERENCES}

1. Anderson JM, Rodriguez A, Chang DT. Foreign body reaction to biomaterials. Semin Immunol. 2008; 20(2): 86-100, doi: 10.1016/j.smim.2007.11.004, indexed in Pubmed: 18162407.

2. Biefer HR, Hürlimann D, Grünenfelder J, et al. Generator pocket adhesions of cardiac leads: classification and correlation with transvenous lead extraction results. Pacing Clin Electrophysiol. 2013; 36(9): 1111-1116, doi: 10.1111/ /pace.12184, indexed in Pubmed: 23713912.

3. Bongiorni MG, Proclemer A, Dobreanu D, et al. Preferred tools and techniques for implantation of cardiac electronic devices in Europe: results of the European Heart Rhythm Association survey. Europace. 2013; 15(11): 1664-1668, doi: 10.1093/europace/eut345, indexed in Pubmed: 24170423.

4. DE Sensi F, Miracapillo G, Cresti A, et al. Pocket Hematoma: A Call for Definition. Pacing Clin Electrophysiol. 2015; 38(8): 909-913, doi: 10.1111/pace.12665, indexed in Pubmed: 25974662.

5. Krupa W, Kozłowski D, Derejko P, et al. Permanent cardiac pacing and its influence on tricuspid valve function. Folia Morphol. 2001; 60(4): 249-257, indexed in Pubmed: 11770335.

6. Mandal S, Pande A, Kahali D. A Rare Case of Very Early Pacemaker Twiddler's Syndrome. Heart Views. 2012; 13(3): 114-115, doi: 10.4103/1995-705X.102157, indexed in Pubmed: 23181182.

7. Martin P, Nunan R. Cellular and molecular mechanisms of repair in acute and chronic wound healing. Br J Dermatol. 2015; 173(2): 370-378, doi: 10.1111/bjd.13954, indexed in Pubmed: 26175283.

8. Maytin M. Device Pocket Scar Predicts Transvenous Lead Extraction Difficulty. J Innovat Cardiac Rhythm Management. 2015; 6(11), doi:10.19102/icrm.2015.061101.

9. Perry L, Karp F, Hauch K, et al. Explanted pacemakers: observations of the long-term foreign body response. J Und Res Bioeng. 2007; 7: 13-21.

10. Rajappan K. Permanent pacemaker implantation technique: part I: arrhythmias. Heart. 2009; 95(3): 259-264, doi: 10.1136/hrt.2007.132753, indexed in Pubmed: 19144885.

11. Rohacek M, Baddour LM. Cardiovascular implantable electronic device infections: associated risk factors and prevention. Swiss Med Wkly. 2015; 145: w14157, doi: 10.4414/ smw.2015.14157, indexed in Pubmed: 26230056.

12. Wynn TA. Cellular and molecular mechanisms of fibrosis. J Pathol. 2008; 214(2): 199-210, doi: 10.1002/path.2277, indexed in Pubmed: 18161745.

13. Velnar T, Bailey T, Smrkolj V. The wound healing process: an overview of the cellular and molecular mechanisms. J Int Med Res. 2009; 37(5): 1528-1542, doi: 10.1177/1 47323000903700531, indexed in Pubmed: 19930861. 